\title{
Shear margins in glaciers and ice sheets
}

\author{
CHARLES RAYMOND \\ Geophysics Program Box 351650, University of Washinglon, Seallle, Washington 98195-1650, U.S.A.
}

\begin{abstract}
Analytical and numerical techniques are used to examine the flow response of a sloped slab of power-law fluid (power $n$ ) subjected to basal boundary conditions that vary spatially across the flow direction, as for example near an icestream margin with planar basal topography. The primary assumption is that basal shear stress is proportional to the basal speed times a spatially variable slip resistance. The ratio of mean basal speed to the speed originating from shearing through the thickness, denoted as $r$, gives a measure of how slippery the bed is. The principal conclusion is that a localized disturbance in slip resistance affects the basal stress and speed in a zone spread over a greater width of the flow. In units of ice thickness $H$, the spatial scale of spreading is proportional to a single dimensionless number $R_{n} \equiv(r / n+1)^{1 / n+1}$ derived from $n$ and $r$. The consequence for a shear zone above a sharp jump in slip resistance is that the shearing is spread out over a boundary layer with a width proportional to $R_{n}$. For an ice stream caused by a band of low slip resistance with a half-width of $w H$, the margins influence velocity and stress in the central part of the band depending on $R_{n}$ in comparison to $w$. Three regimes can be identified, which for $n=3$ are quantified as follows: low $r$ defined as $R_{3}<0.1 \mathrm{w}$, for which the central flow is essentially unaffected by the margins and the driving stress is supported entirely by basal drag; high $r$ defined as $R_{3}>1 \mathrm{w}$, for which the boundary layers from both sides bridge across the full flow width and the driving stress in the center is supported almost entirely by side drag; intermediate $r$, for which the driving stress in the center is supported by a combination of basal and side drag. Shear zones that are narrower than predicted on the basis of this theory $\approx R_{3}$ ) would require localized softening of the ice to explain the concentration of deformation at a shorter scale.
\end{abstract}

\section{INTRODUCTION}

Velocity and stress vary across the widths of valley glaciers (Paterson, 1994). The shape of the cross-section of the valley exerts an important influence, which has been analyzed extensively assuming a no-slip boundary condition (Nye, 1965). Both observations (Raymond, 1971 ) and models (Reynaud, 1973; Harbor, 1992) have shown that variations in the basal-slip condition across the width of a glacier can also significantly affect the pattern of shearing in the ice.

Cross-flow effects arising from the basal-slip condition as opposed to basal topography are perhaps best exemplified by ice streams, such as those that flow from the interior of the West Antarctic ice sheet to the Ross Ice Shelf. These ice streams are fast currents of ice that are embedded in slower-moving ice. In some cases the ice thickness is nearly constant and there is no clear topographic control of the position of the boundaries between the fast- and slow-moving ice Shabtaie and Bentley, 1987). In these cases, the ice streams owe their existence to strong contrasts in slip resistance at the ice base that allow rapid basal motion under the ice streams and not outside their margins (Bentley, 1987). In addition, there may be variations in slip resistance within ice streams that are unrelated to basal topography
MacAyeal, 1992). The stress and velocity distributions in the ice and their manifestations as variations in internal temperature and morphology of the ice surface are strongly affected by the extension of effects originating at the bed upward through the ice thickness. The shear zones at the edges of ice streams are dramatic examples.

Effects from cross-flow variations in slip resistance have not been systematically explored from the point of view of glacier mechanics. This paper provides some initial steps in that direction through two principal purposes. The first purpose is to characterize in a general way how a cross-flow variation in slip resistance is transferred through the thickness to the surface. This goal is accomplished with a simplified analytical model that allows small but otherwise arbitrary variations in slip resistance from a mean condition and assumes linear viscous behavior for the ice. The second purpose is to examine the specific case of a shear zone arising from a sharp jump in slip resistance, such as may occur at an icestream margin. This necessitates numerical calculations that accommodate large variations in slip resistance and account for non-linear flow law for the ice.

To isolate effects from cross-flow variations in slip condition as opposed to bed elevation, a planar, laterally horizontal bed geometry is assumed. Longitudinal variations in geometry and slip condition are excluded. 
Variations in temperature or ice fabric that could influence the flow (e.g. Echelmeyer and others, 1994) are not considered. Although the analysis is idealized, it identifies important features of the theoretical flow pattern that would remain as effects from topography, temperature and fabric are taken into account.

Foremost amongst these features is the way in which a change in the resistance to slip at a given location affects the basal velocity and stress over a wider area. The spatial scale of this delocalization is related to the relative contributions to the total surface motion from basal slip and shearing within the ice. The spatial spreading and related smoothing have general implications for the manner in which effects from basal processes are transferred to the upper surface. There are corresponding implications for the inversion of observations of cross-flow variations in surface velocity for the bed conditions that produce them. For the specific case of a sharp jump in resistance to slip, the scale of the spreading determines the width of a marginal boundary layer within which stress propagation from the side has a significant influence on the flow.

\section{THEORETICAL FRAMEWORK}

\section{Geometry, kinematic and flow-law assumptions}

My purpose is to calculate the distribution of ice velocity and stress as a function of position in a cross-section perpendicular to the flow direction, supposing that the basal boundary condition varies across the flow direction but not parallel to this direction. To do the analysis I will use both dimensional variables denoted by lower-case letters with a hat $(\hat{)})$ and corresponding non-dimensional ones (denoted by un-hatted lower-case letters) that are related through scales as defined in the text and listed in Table 1. Figure 1 shows the assumed geometry of the ice. With reference to the definitions of coordinates in Figure 1 and notation in Table $1, I$ assume that the flow is

Table 1. List of symbols

\begin{tabular}{|c|c|c|c|c|}
\hline $\begin{array}{l}\text { Dimensional } \\
\text { variable }\end{array}$ & $\begin{array}{c}\text { Non-dimensional } \\
\text { variable }\end{array}$ & $\begin{array}{l}\text { Dimensional } \\
\text { scale }\end{array}$ & Description & Definition \\
\hline$\hat{\mathbf{g}}$ & & & Gravitational acceleration & \\
\hline$\hat{\rho}$ & & & Firn/ice density & \\
\hline$H$ & 1 & {$[H]$} & Ice thickness & Figure 1 \\
\hline$T$ & 1 & {$[T]$} & Driving stress & Equation (7a) \\
\hline$U_{\mathrm{D}}$ & 1 & {$\left[U_{\mathrm{D}}\right]$} & Deformational speed & Equation $(7 \mathrm{~b})$ \\
\hline \multirow[t]{2}{*}{$\Xi$} & 1 & {$[\Xi]$} & Slip resistance scale & Equation 5 \\
\hline & $r$ & & Slip ratio & Equation $(8)$ \\
\hline$\hat{\eta}$ & & & Effective viscosity & Equation (1b) \\
\hline \multirow[t]{3}{*}{$B$} & $2^{-1+1 / n}(n+1)^{-1 / n}$ & & Flow-law hardness & Equation (1b) \\
\hline & $n$ & & Flow-law power & Equation $1 \mathrm{~b})$ \\
\hline & $m$ & & Power in slip law & Equation $(36)$ \\
\hline$\hat{\sigma}$ & & & Stress tensor & \\
\hline$\hat{\tau}$ & & & Deviatoric stress tensor & Equation (1a) \\
\hline$\hat{\mathbf{d}}$ & & & Strain-rate tensor & Equation ( $1 \mathrm{a})$ \\
\hline$\hat{x}$ & $x$ & {$[H]$} & Downslope distance & Figure 1 \\
\hline$\hat{y}$ & $y$ & {$[H]$} & Cross-slope distance & Figure 1 \\
\hline$\hat{z}$ & $z$ & {$[H]$} & Slope normal distance & Figure 1 \\
\hline$\hat{w}$ & $w$ & {$[H]$} & Stream half-width & \\
\hline$\hat{l}$ & l & {$[H]$} & Boundary-layer width & Equation $(32)$ \\
\hline$\hat{u}(\hat{y}, \hat{z})$ & $u(y, z)$ & {$\left[U_{\mathrm{D}}\right]$} & Downslope speed & Figure 1, Equation (7) \\
\hline$\hat{u}_{\mathrm{s}}(\hat{y})$ & $u_{\mathrm{s}}(y)$ & {$\left[U_{\mathrm{D}}\right]$} & Surface speed & $u_{\mathrm{s}}(y)=u(y, 0)$ \\
\hline \multirow[t]{2}{*}{$\hat{u}_{\mathrm{b}}(\hat{y})$} & $u_{\mathrm{b}}(y)$ & {$\left[U_{\mathrm{D}}\right]$} & Basal speed & $u_{\mathrm{b}}(y)=u(y, 1)$ \\
\hline & $u^{*}(y, z)$ & {$\left[U_{\mathrm{D}}\right]$} & Speed departure & Equation (13c) \\
\hline \multirow[t]{2}{*}{$\hat{e}(\hat{y}, \hat{z})$} & $e(y, z)$ & {$\left[U_{\mathrm{D}} / H\right]$} & Effective strain rate & Equations (2c) and (9c) \\
\hline & $e_{\mathrm{m}}$ & {$\left[U_{\mathrm{D}} / H\right]$} & Maximum shear rate & Equation $(29)$ \\
\hline$\hat{t}_{y}(\hat{y}, \hat{z})$ & $t_{y}(y, z)$ & {$[T]$} & Side shear stress & Equations (2a), (7a), 9a) \\
\hline \multirow{4}{*}{$\begin{array}{c}\hat{t}_{z}(\hat{y}, \hat{z}) \\
\hat{t}_{\mathrm{b}}(\hat{y})\end{array}$} & $t_{z}(y, z)$ & {$[T]$} & Slope shear stress & Equations $(2 b),(7 a),(9 b)$ \\
\hline & $t_{\mathrm{b}}(y)$ & {$[T]$} & Basal shear stress & $t_{\mathrm{b}}(y)=-t_{z}(y, 1)$ \\
\hline & $t_{y}{ }^{*}(y, z)$ & {$[T]$} & Stress departure & Equation (13b) \\
\hline & $t_{z}^{*}(y, z)$ & {$[T]$} & Stress departure & Equation (13a) \\
\hline \multirow[t]{5}{*}{$\hat{\xi}(\hat{y})$} & $\xi(y)$ & {$[\Xi]$} & Slip resistance & Equation $(5)$ \\
\hline & $\xi^{*}(y)$ & {$[\Xi]$} & Slip-resistance departure & Equation $(12)$ \\
\hline & $\tilde{F}_{\mathrm{u}}\left(F_{\mathrm{u}}\right)$ & & Velocity transfer & Equations (2l) and (26a) \\
\hline & $\tilde{F}_{\mathrm{t}}\left(F_{\mathrm{t}}\right)$ & & Stress transfer & Equations (23) and (26b) \\
\hline & $\tilde{F}_{\mathrm{sl}}\left(F_{\mathrm{sl}}\right)$ & & Slip transfer & Equation $(24)$ \\
\hline
\end{tabular}




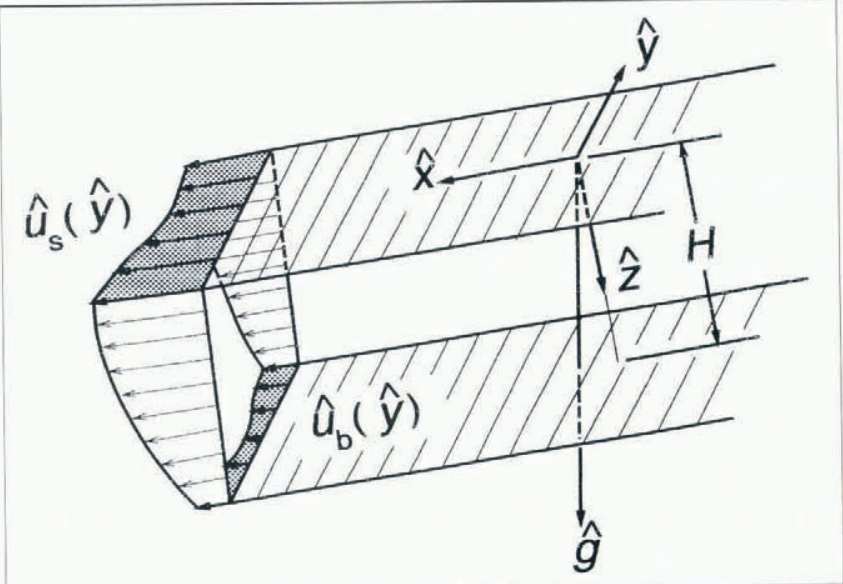

Fig. 1. Schematic of geometry and coordinate system.

non-accelerating and rectilinear, such that ice moves only in the downslope direction $(\hat{x})$ at a speed $\hat{u}$ that depends on $\hat{y}$ and $\hat{z}$, but not on $\hat{x}$ or time. Since the assumed motion is parallel to the upper $(\hat{z}=0)$ and lower $(\hat{z}=H)$ surfaces, the geometry is independent of time.

To describe the ice, I assume constant density $\hat{\rho}$ and a quasi-viscous flow law relating deformation rate $\hat{\mathbf{d}}$ and deviatoric stress $\hat{\tau}$ by

$$
\hat{\tau}=2 \hat{\eta} \hat{\mathbf{d}}
$$

where $\hat{\eta}$ is an effective viscosity. To account for non-linear flow law, $\hat{\eta}$ may depend on stress or deformation rate. A power relation between deformation rate and stress may be expressed by

$$
\hat{\eta}(\hat{e})=B \hat{e}^{-(1-1 / n)}
$$

where $n$ and $B$ are constants and $\hat{e}^{2}=0.5 \hat{\mathbf{d}}: \hat{\mathbf{d}}$ is an effective shear-strain rate related to the second invariant of $\hat{\mathbf{d}}$. When $n=1$, Equation ( $1 \mathrm{~b}$ ) gives $\hat{\eta}=B$, which corresponds to a Newtonian fluid. It is common to assume $n=3$ for ice (Paterson, 1994). By assuming $B$ is constant, I neglect influences on the ice deformation that could come from variations in temperature or fabric.

These assumptions have some immediate implications that simplify the mathematical problem (e.g. Nye, 1965). Mass is conserved automatically, since the rectilinear flow pattern is divergenceless and density is constant. The only non-zero deformation-rate components are $2 \hat{d}_{x y}=\partial \hat{u} / \partial \hat{y}$ and $2 \hat{d}_{x z}=\partial \hat{u} / \partial \hat{z}$. Thus, by Equation (1) the only nonzero shear-stress components are $\hat{\tau}_{x y}=\hat{t}_{y}$ and $\hat{\tau}_{x z}=\hat{t}_{z}$, and these are independent of position along the flow. Conservation of momentum in the cross-flow directions $\hat{y}$ and $\hat{z}$ is then satisfied trivially by a hydrostatic distribution of normal-stress components $\left\langle\hat{\sigma}_{x x}=\hat{\sigma}_{y y}=\right.$ $\hat{\sigma}_{z z}=-\hat{\rho} \hat{g}_{z} z$ ). The mathematical problem is then reduced to finding $\hat{t}_{y y}(\hat{y}, \hat{z}), \hat{t}_{z}(\hat{y}, \hat{z})$ and $\hat{u}(\hat{y}, \hat{z})$ subject to the flow law and conservation of momentum in the flow direction $\hat{x}$. Since the effect of temperature on the flow law is neglected, it is not necessary to consider conservation of energy.

If the flow law were to have a more complex nonlinearity than allowed by Equations (1), then the simplifications of planar geometry and rectilinear flow could break down. For example, a flow law with "normal stress effects" (such that corresponding components of deviatoric stress and deformation rate are not proportional) could cause a more complex balance of momentum in the $y$ and $z$ directions, force cross-flow secondary motions and/or displace the upper surface to a non-planar configuration. Normal stress effects are not commonly assumed for ice (Paterson, 1994), and I will not introduce that complexity here.

\section{Field equations and boundary conditions}

With the simplifications discussed above, the flow law and conservation of momentum reduce to

$$
\begin{gathered}
\hat{t}_{y}=B \hat{e}^{-(1-1 / n)} \frac{\partial \hat{u}}{\partial \hat{y}} \\
\hat{t}_{z}=B \hat{e}^{-(1-1 / n)} \frac{\partial \hat{u}}{\partial \hat{z}} \\
\hat{e}=\frac{1}{2}\left[\left(\frac{\partial \hat{u}}{\partial \hat{y}}\right)^{2}+\left(\frac{\partial \hat{u}}{\partial \hat{z}}\right)^{2}\right]^{\frac{1}{2}} \\
\frac{\partial \hat{t}_{y}}{\partial \hat{y}}+\frac{\partial \hat{t}_{z}}{\partial \hat{z}}+\hat{\rho} \hat{g}_{x}=0 .
\end{gathered}
$$

Equations (2) and (3) are the field equations to determine $\hat{t}_{y}(\hat{y}, \hat{z}), \hat{t}_{z}(\hat{y}, \hat{z}), \hat{u}(\hat{y}, \hat{z})$.

The upper surface $(\hat{z}=0)$ is shear-stress free, so that

$$
\hat{t}_{z}(\hat{y}, 0)=0 \text {. }
$$

At the base $(\hat{z}=H)$, the shear stress acting on the bed $\hat{t}_{\mathrm{b}}(y)=-\hat{t}_{z}(\hat{y}, H)$ and the basal speed $\hat{u}_{\mathrm{b}}(\hat{y})=\hat{u}(\hat{y}, H)$ are assumed to be related by

$$
\hat{t}_{\mathrm{b}}(\hat{y})=\hat{\xi}(\hat{y}) \hat{u}_{\mathrm{b}}(\hat{y})
$$

where $\hat{\xi}$ describes a resistance to slip.

For my purpose it is not necessary to distinguish between basal slip that arises from shearing in soft subglacial material, sliding associated with a discrete velocity discontinuity at the base of the ice or some combination. Either kind of process can be represented by Equation (4b). By allowing $\hat{\xi}$ to depend on $\hat{u}_{\mathrm{b}}$, Equation $(4 \mathrm{~b})$ can be used to represent non-linear relationships between $\hat{t}_{\mathrm{b}}$ and $\hat{u}_{\mathrm{b}}$, as in various theoretical slip laws that have been proposed. Although realistic relationships between $t_{\mathrm{b}}$, and $u_{\mathrm{b}}$ are likely to be non-linear, the details of such nonlinearity are unknown. Therefore, in most of the development, I consider only linear relationships subject to the requirement that $t_{\mathrm{b}}$ increases as $u_{\mathrm{b}}$ increases $(\hat{\xi}>0)$. The view I pursue here is that $\hat{\xi}$ depends on $\hat{y}$ because control variables, such as bed roughness, till thickness, till composition, temperature and/or water pressure, vary with $\hat{y}$ and alter the way $\hat{u}_{\mathrm{b}}$ and $\hat{t}_{\mathrm{b}}$ are related laterally across the bed. At the end of the paper I consider the implications of non-linear slip laws.

\section{Dimensionless formulation}

I describe the slip resistance $\hat{\xi}(\hat{y})$ as 


$$
\hat{\xi}(\hat{y})=\Xi \xi(\hat{y})
$$

where $\Xi$ is a constant that sets the scale of $\hat{\xi}(\hat{y})$, and $\xi(\hat{y})$ is a dimensionless description of the spatial pattern of crossflow variation.

For the condition that $\xi(\hat{y})=1$ in Equation (5) that is, $\hat{\xi}(\hat{y})$ is constant at value $\Xi)$, Equations $(2)-(4)$ lead straightforwardly to the one-dimensional solution

$$
\begin{aligned}
& \hat{t}_{z}(\hat{y}, \hat{z})=-T \frac{\hat{z}}{H} \\
& \hat{t}_{y}(\hat{y}, \hat{z})=0 \\
& \hat{u}(\hat{y}, \hat{z})=U_{\mathrm{D}}\left[1-(\hat{z} / H)^{n+1}\right]+T / \Xi
\end{aligned}
$$

where

$$
T=\hat{\rho} \hat{g}_{x} H
$$

and

$$
U_{\mathrm{D}}=\frac{2^{(1-n)}}{(n+1) B^{n}} T^{n} H .
$$

This solution describes a planar slab slipping over its base at a uniform rate. It is well known in the theory of fluid flow. For a non-slipping Newtonian fluid $n=1, B=\eta$. $\Xi=\infty)$, it predicts a parabolic velocity profile Batchelor, 1967). It is widely used normally with $n=3$ to approximate flow of glaciers (Paterson, 1994). Equations (6) show $T$ to be the basal shear stress $\hat{t}_{\mathrm{b}}=-\hat{t}_{z}(\hat{y}, H), U_{\mathrm{D}}$ to be the total deformational speed arising from shearing in the ice over the thickness $H$, and $T / \Xi$ to be a uniform rate of slip at the base.

The quantities $T, U_{\mathrm{D}}$ and $\Xi$ can be defined more generally from Equations (5) and (7) independently of the particular spatial variation of $\xi(\hat{y})$. The stress $T$ is commonly termed the driving stress, from which the actual $\hat{t}_{\mathrm{b}}$ may depart when $\xi$ depends on $\hat{y}$. I use $H, T, U_{\mathrm{D}}$ and $\Xi$ as scales for length, shear stress, speed and slip resistance to define dimensionless variables listed in Table 1.

To describe the relative contributions of basal slip $T / \Xi$ and internal deformation $U_{\mathrm{D}}$ to the motion, I define the slip ratio

$$
r=T / \Xi U_{\mathrm{D}} .
$$

With these definitions of scales and related dimensionless variables (Table 1), the field Equations (2) and (3) and boundary conditions (4) reduce to

$$
\begin{gathered}
t_{y}=\frac{1}{2}\left(\frac{2}{n+1}\right)^{\frac{1}{n}} \mathrm{e}^{-\left(1-\frac{1}{n}\right)} \frac{\partial u}{\partial y} \\
t_{z}=\frac{1}{2}\left(\frac{2}{n+1}\right)^{\frac{1}{n}} \mathrm{e}^{-\left(1-\frac{1}{n}\right)} \frac{\partial u}{\partial z} \\
e=\frac{1}{2}\left[\left(\frac{\partial u}{\partial y}\right)^{2}+\left(\frac{\partial u}{\partial z}\right)^{2}\right]^{\frac{1}{2}} \\
\frac{\partial t_{y}}{\partial y}+\frac{\partial t_{z}}{\partial z}+1=0 \\
\partial u(y, 0) / \partial z=0
\end{gathered}
$$

$$
t_{\mathrm{b}}(y)=\xi(y) u_{\mathrm{b}},(y) / r .
$$

Two parameters, the flow-law power $n$ and the slip ratio $r$, appear in the non-dimensional equations. For the flow law, I will restrict attention to $n=1$ (which allows simple analytical solution) and $n=3$ (which corresponds to realistic ice). The slip ratio $r$ can in principle have an enormous range from 0 no slip) to $\infty$ (frictionless base). A principal goal of this paper is to explore how the size of $r$ affects the character of the the solutions to Equations (9)-(11).

\section{GENERAL TRANSFER GHARACTERISTICS}

\section{Equations for Newtonian viscous deformation}

With the simplification of Newtonian viscosity $n=1$ and the constraint that fluctuations in the slip resistance $\xi(z)$ are small compared to mean conditions, it is possible to find an analytical solution to Equations $9-11$ for an otherwise arbitrary distribution of $\xi(z)$. For the analysis it is convenient to express the distributions of slip resistance, speed and stress as

$$
\begin{gathered}
\xi(y)=1+\xi^{*}(y) \\
t_{z}(y, z)=t_{z}^{*}(y, z)-z \\
t_{y y}=t_{y}{ }^{*}(y, z) \\
u(y, z)=(1-y)^{2}+r+u^{*}(y, z) .
\end{gathered}
$$

When $\xi^{*}=t_{y}{ }^{*}=t_{z}{ }^{*}=u^{*}=0$, then Equations 13 are the solution to Equations 9 and $(10)$, with $\xi(y)=1$ (uniform slip), and are dimensionless representations of Equations 6 . The starred quantities $t_{l j}{ }^{*} t_{2}{ }^{*}$ and $u^{*}$ represent departures of stress and speed that arise when $\xi^{*}(y) \neq 0$.

Introduction of Equations (13) into Equations 9 with $n=1$ gives

$$
\begin{aligned}
& t_{z}{ }^{*}=\frac{1}{2} \frac{\partial u^{*}}{\partial z} \\
& t_{y}{ }^{*}=\frac{1}{2} \frac{\partial u^{*}}{\partial y}
\end{aligned}
$$

and then into Equation 10$)$ gives

$$
\frac{\partial^{2} u^{*}(y, z)}{\partial y^{2}}+\frac{\partial^{2} u^{*}(y, z)}{\partial z^{2}}=0 .
$$

Substitution of Equations (12) and (13) into Equation (11) gives the boundary conditions

$$
\frac{\partial u^{*}(y, 0)}{\partial z}=0
$$

and

$$
t_{\mathrm{b}}{ }^{*}(y)=\xi^{*}(y)+\frac{u_{\mathrm{b}}{ }^{*}(y)}{r}+\frac{\xi^{*}(y) u_{\mathrm{b}}{ }^{*}(y)}{r}
$$

where $u_{\mathrm{b}}{ }^{*}=u_{\mathrm{b}},(y)-r$ and $t_{\mathrm{b}}{ }^{*}(y)=t_{\mathrm{b}}(y)-1$.

The approximation in the analytical solution is to 
assume that $\xi^{*} \ll 1$ and $u_{\mathrm{b}}{ }^{*} \ll r$, which holds when the variations in $\xi$ and $u_{\mathrm{b}}$ are small in comparison to their means. With this assumption, the third (non-linear) term on the right of Equation (16b) can be neglected.

\section{Approximate solution}

The solution for $u^{*}, t_{y}{ }^{*}$ and $t_{z}{ }^{*}$ when $\xi^{*} \neq 0$ can be sought using Fourier-transform methods in the form

$$
\tilde{f}(\nu, z)=\int_{-\infty}^{+\infty} f(y, z) \exp (-2 \pi i \nu y) \mathrm{d} y
$$

with inverse

$$
f(y, z)=\int_{-\infty}^{+\infty} \tilde{f}(\nu, z) \exp (+2 \pi i \nu y) \mathrm{d} \nu .
$$

In this formulation the transform variable $\nu$ is wave number described by inverse wavelength.

The momentum Equation (15) when transformed becomes

$$
0=\frac{\partial^{2} \tilde{u}^{*}(\nu, z)}{\partial z^{2}}-(2 \pi \nu)^{2} \tilde{u}^{*}(\nu, z)
$$

This has exponential solutions $\exp (-2 \pi \nu z)$ and $\exp$ $(+2 \pi \nu z)$. The boundary condition on the upper surface Equation (16a)) requires that these exponentials be be combined in the form

$$
\tilde{u}^{*}(\nu, z)=A(\nu) \cosh (2 \pi \nu z)
$$

where $A(\nu)$ is to be determined from the boundary condition at the base.

At this point several features of the solution can be identified that will be useful later. The speed on the upper surface is given by

$$
\tilde{u}_{\mathrm{s}}^{*}(\nu)=\tilde{u}^{*}(\nu, 0)=A(\nu) .
$$

The basal speed is given by

$$
\tilde{u}_{\mathrm{b}}{ }^{*}(\nu)=\tilde{u}^{*}(\nu, 1)=A(\nu) \cosh (2 \pi \nu)
$$

Equations (20) show that the surface and basal velocities are related by

$$
\tilde{u}_{\mathrm{s}}^{*}(\nu)=\tilde{F}_{\mathrm{u}}(\nu) \tilde{u}_{\mathrm{b}}{ }^{*}(\nu)
$$

where

$$
\tilde{F}_{\text {u }}(\nu)=\frac{1}{\cosh (2 \pi \nu)}=\operatorname{sech}(2 \pi \nu) .
$$

Basal shear stress $\tilde{t}_{\mathrm{b}}{ }^{*}(\nu)$ can be calculated from Equations (14a) and (19) as

$$
\tilde{t}_{\mathrm{b}}^{*}(\nu)=-\tilde{t}_{z}^{*}(\nu, 1)=-A(\nu) \pi \nu \sinh (2 \pi \nu) .
$$

Comparison of Equations (20b) and (22) shows that

$$
\tilde{t}_{\mathrm{b}}^{*}(\nu)=\tilde{F}_{\mathrm{t}}(\nu) \tilde{u}_{\mathrm{b}}{ }^{*}(\nu)
$$

where

$$
\tilde{F}_{\mathrm{t}}(\nu)=-\pi \nu \tanh (2 \pi \nu)
$$

To complete the solution it is necessary to satisfy the basal boundary condition as described by Equation (16b) in the approximation of neglecting the non-linear term. Substitution of Equation (23a) into Equation (16b) gives

$$
\tilde{u}_{\mathrm{b}}{ }^{*}(\nu)=\tilde{F}_{\mathrm{sl}}(\nu) \tilde{\xi}^{*}(\nu)
$$

where

$$
\tilde{F}_{\mathrm{sl}}(\nu)=-\frac{r}{1-r \tilde{F}_{\mathrm{t}}(\nu)}
$$

With these results the solution in the wave number domain for $u_{\mathrm{b}}{ }^{*}, t_{\mathrm{b}}{ }^{*}$ and $u_{\mathrm{s}}{ }^{*}$ resulting from a specified $\xi^{*}$ can be summarized as follows:

$$
\begin{gathered}
\tilde{u}_{\mathrm{b}}{ }^{*}(\nu)=\tilde{F}_{\mathrm{sl}}(\nu) \tilde{\xi}^{*}(\nu) \\
\tilde{t}_{\mathrm{b}}{ }^{*}(\nu)=\tilde{F}_{\mathrm{t}}(\nu) \tilde{u}_{\mathrm{b}}{ }^{*}(\nu)=\tilde{F}_{\mathrm{t}}(\nu) \tilde{F}_{\mathrm{sl}}(\nu) \tilde{\xi}^{*}(\nu) \\
\tilde{u}_{\mathrm{s}}^{*}(\nu)=\tilde{F}_{\mathrm{u}}(\nu) \tilde{u}_{\mathrm{b}}{ }^{*}(\nu)=\tilde{F}_{u}(\nu) \tilde{F}_{\mathrm{sl}}(\nu) \tilde{\xi}^{*}(\nu)
\end{gathered}
$$

where the the $\tilde{F}$ are transfer functions given by Equations (21b), (23b) and (24b). The transfer functions are plotted in Figure 2. The solution in the wave-number domain for the full depth distribution of velocity can be found from Equation (19), using Equations (25c) and (20a) to determine $A(\nu)$.
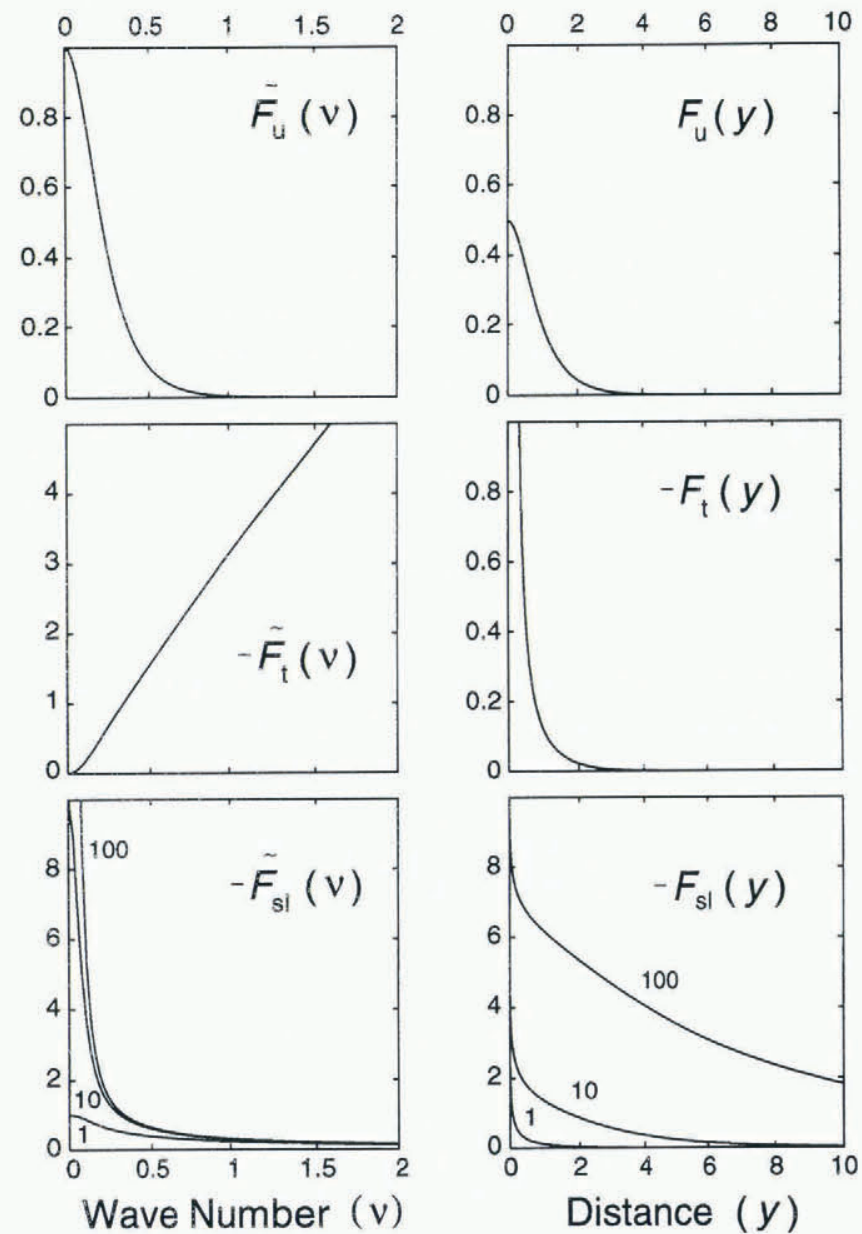

Fig. 2. Wave-number and space representations of transfer functions for linear flow defined in Equations (21), (23) and (24). Numbers on curves for $F_{\mathrm{s} I}$ give values of slip ratio $r$. 
The spatial forms of the transfer functions $\tilde{F}_{11}(\nu)$ and $\tilde{F}_{t}(\nu)$ can be expressed analytically as

$$
F_{\mathrm{u}}(y)=\frac{1}{2} \operatorname{sech}\left(\frac{\pi y}{2}\right)
$$

and

$$
F_{\mathrm{t}}(y)=-\frac{1}{4} \operatorname{coth}\left(\frac{\pi y}{2}\right) \operatorname{csch}\left(\frac{\pi y}{2}\right) .
$$

These expressions can be found from Equations (21b) and (23b) with standard manipulations using similarity, the derivative theorem and well-known Fourier-transform pairs (Bracewell, 1978). I have not found an analytical expression for the spatial form of $F_{\mathrm{sl}}(y)$ from $\tilde{F}_{\mathrm{sl}}(\nu)$, but it can be found numerically using the discrete Fourier transform. Figure 2 shows the spatial forms of $F_{14}(y), F_{\mathrm{t}}(y)$ and $F_{s l}(y)$. They can be viewed as space-domain filters that can be convolved with $\xi^{*}(y)$ and $u_{\mathrm{b}}{ }^{*}(y)$ to define the solution in the space domain in a way alternative to the convolution in the wave-number domain defined by Equations (25).

Important general properties of the solution are displayed by the correlations amongst $\xi^{*}(y), u_{\mathrm{b}}{ }^{*}(y)$ and $t_{b}{ }^{*}(y): u_{b}{ }^{*}(y)$ and $\xi^{*}(y)$ are negatively correlated Equations (24); Fig. $2 ; t_{\mathrm{b}}{ }^{*}(y)$ and $u_{\mathrm{b}}{ }^{*}(y)$ are negatively correlated Equations (23); Fig. 2). Thus, $u_{\mathrm{b}}{ }^{*}(y)$ is low and $t_{1}{ }^{*}(y)$ is high where $\xi^{\star}(y)$ is high (i.e. bed is sticky).

\section{Delocalization of slip-resistance response}

The functions $F_{\mathrm{s}}, F_{\mathrm{t}}$ and $F_{\mathrm{t}}$ describe how departures in slip resistance from a reference level affect the distributions of velocity and stress. The effect can be viewed in terms of spectral $(\tilde{F}(\nu, z))$ or spatial $(F(y, z))$ characteristics. I shall now focus on the spatial characteristics from the point of view of how a localized disturbance in slip resistance affects the velocity and stress on the base and in the ice over a broader width spread across the flow. The widths of the spatial forms of $F$ give measures of the delocalization.

The widths of $F$ can be defined analytically in terms of the second moment

$$
\left[y^{2}\right]=\int_{-x}^{+x} y^{2} F(y) \mathrm{d} y .
$$

It can be evaluated in wave number domain from

$$
\left[y^{2}\right]=\frac{1}{4 \pi^{2} \tilde{F}(\nu)} \frac{\partial^{2} \tilde{F}(\nu)}{\partial \nu^{2}}
$$

evaluated at $\nu=0$ Bracewell, 1978), which from Equations (2lb), (23b) and (24b) gives

$$
\begin{aligned}
& {\left[y^{2}\right]_{\mathrm{U}}=1} \\
& {\left[y^{2}\right]_{\mathrm{t}}=1} \\
& {\left[y^{2}\right]_{\mathrm{H}}=\sqrt{r} .}
\end{aligned}
$$

These spatial widths are apparent in Figure 2. The widths of $F_{\mathrm{u}}$ and $F_{\mathrm{t}}$ are scaled by thickness $[H]$ and are otherwise independent of the flow dynamics. The width of $F_{\text {si }}$ depends on the flow dynamics through the slip ratio $r$. Implications of these general characteristics can be illustrated with some specilic examples.
Figure 3a shows the solution for an impulse in $\xi^{*}(y)=-\delta(y)$ for the case $r=10$. The solution for $u_{\mathrm{b}}{ }^{*}$ is given directly from the transfer function $F_{s]}$ (Fig. 2). The basal speed $u_{\mathrm{b}}{ }^{*}$ is spread out over a half-width of about 3, which corresponds to the width of $F_{\mathrm{s}}$ and is consistent with the $\sqrt{r}$ dependence for the width of $F_{\mathrm{sl}}$ (Equation $28 \mathrm{c}$ )). The surface speed $u_{\mathrm{s}}{ }^{*}$ and base stress $\iota_{\mathrm{b}}{ }^{*}$ show only slight additional spreading associated with the unit widths of $F_{\mathrm{u}}$ and $F_{\mathrm{t}}$.
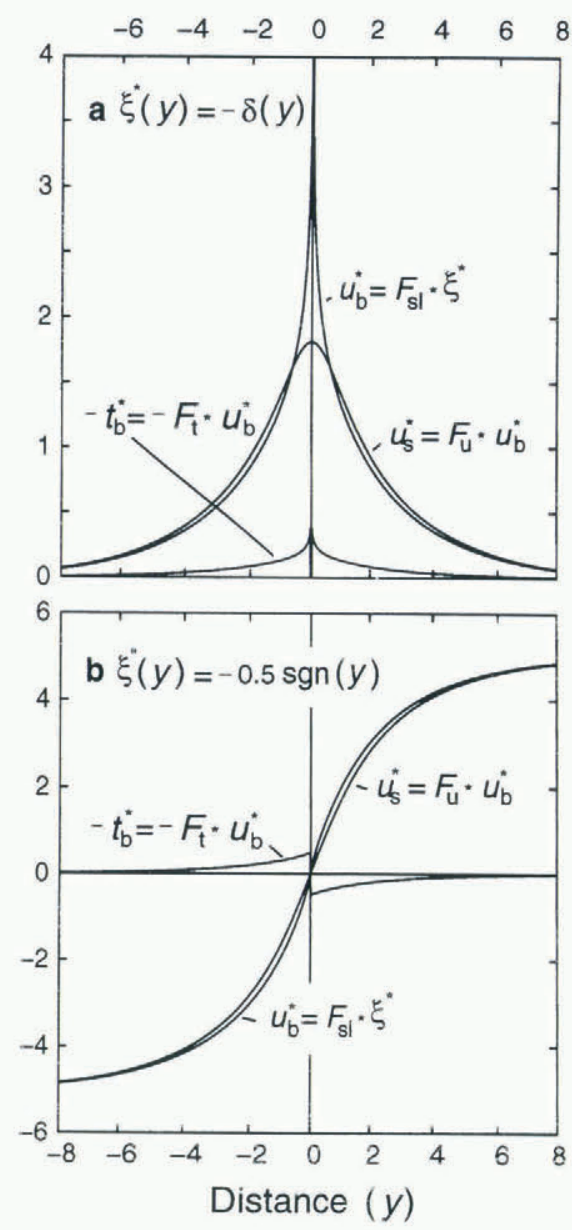

Fig. 3. Solution for the departures in bed speed $u_{1}{ }^{*}$, basal shear stress $t_{1},{ }^{*}$ and surface speed $u_{\mathrm{s}}{ }^{*}$ caused by a della function impulse (a) and a slep function (b) in the slip resistance $\xi$.

Figure $3 b$ shows results for a jump in $\xi^{*}(y)=$ $-0.5 \operatorname{sgn}(y)$ for the case $r=10$. The transition in basal speed $u_{\text {b }}$, produces a shear margin that is spread out over a boundary layer with a half-width of about 3, which corresponds to the width of $F_{\mathrm{s}}$ for $r=10$.

These solutions illustrate that the effects of a sticky or slippery zone can be spread out over a multi-thickness width. By reference to $F_{\text {s1 }}$ Equations (24) and 28c); Fig. 2), the width of the spreading is larger, when the bed is more generally slippery in comparison to the deformability of the ice as described by $r$. This means, for example, that at a higher general level of $u_{\mathrm{b}}$, the spatial variation of $u_{\mathrm{b}}$ reflects a more strongly smoothed response to spatial patterns of $\xi$. 


\section{Stress concentration and relaxation at a shear margin}

The results in Figure $3 b$ illustrate some general features of a shear margin caused by a jump in slip resistance. I will refer to the location of the jump in $\xi(y=0)$ as the margin. There is a redistribution of basal shear stress from the slippery to the sticky side of the margin. Two zones of the boundary layer can be defined. On the slippery side $(y>0)$, side drag provides partial support to the ice column and $t_{\mathrm{b}}<1$. On the sticky side $(y<0), t_{\mathrm{b}}>1$ because ice is being dragged along from the side. I will refer to these zones as the stress-relaxation and stressconcentration zones.

Global force balance requires that the basal shearforce reduction in the relaxation zone, the shear force acting across the margin and the basal shear-force increase in the concentration zone are all equal, which expresses the transfer of driving stress from the relaxation zone through the margin to the concentration zone.

The discontinuous jump in $\xi$ at $y=0$ causes $\partial u / \partial y$ and $t_{y}$ to be singular at the base and $t_{\mathrm{b}}$ to be discontinuous and singular. Thus, the stress relaxation and concentration can be very large locally near the margin.

The boundary layer has reflection symmetry. The margin $(y=0)$ is the location of maximum side-shear strain rate $(\partial u / \partial y)$ and stress $\left(t_{y}\right)$, and the signs of $\partial^{2} u / \partial y^{2}$ and $\partial t_{y} / \partial y$ change across the margin. The marginal speed both at the surface $u_{\mathrm{s}} \equiv u(0,0)$ and at the bed $u_{\mathrm{b}}(0)=u(0,1)$ is the mean of the far field values on either side. The reflection symmetry arises because of the neglect of the non-linear cross-term in the basal-boundary condition Equation (16b)). Although this symmetry is valid for small jumps in $\xi$, it is shown below that it breaks down for large jumps.

\section{SHEAR MARGINS}

\section{Assumptions for numerical solutions}

The approximation of the foregoing solution for a step in $\xi$ requires the step to be small compared to the mean i.e. $\left.\xi^{*} \ll 1\right)$. In reality, there can be large jumps, for example, at ice-stream margins. Solutions for large jumps were found numerically using finite elements. The basal resistance $\xi$ was chosen to be very large (effectively infinite and constant outside of the ice-stream boundary, which suppresses any basal motion. Following current terminology, I refer to this slow ice as ridge ice. The value of $\xi$ was chosen relatively small and constant under the ice stream to allow significant slip rate.

I define the scale for slip resistance $\Xi$ to be the value of $\hat{\xi}$ beneath the ice stream. The ratio $r$ defined by Equation (8) then corresponds to the slip speed in units of $U_{\mathrm{D}}$ that would occur at the base of the ice stream in the presence of the full driving stress of $1[T]$ undiminished by side drag.

The solution was carried out in a region of finite width extending $10[H]$ into the slow-moving ridge ice and $30[H]$ into the fast-moving stream ice. The boundary condition $\partial u / \partial y=0$ was imposed at these lateral boundaries. The distance $10[H]$ into the ridge was sufficient that this edge of the solution region was decoupled from effects at the shear margin. The distance $30[H]$ into the stream flow and the symmetry imposed by the boundary condition correspond to full ice-stream width of $60[H]$. More generally I denote the half-width of the stream as $w$, which is $30[H]$ in these specific numerical calculations.

\section{Results from numerical calculations}

Numerical calculations were done for a sequence of different values of slip ratio $r$ to display the effect of $r$ on the character of the solutions. Results were obtained for $r=1 \times 10^{k}, 2 \times 10^{k}$ and $5 \times 10^{k}$, where $k=1-5$ for $n=1$, and $k=1-7$ for $n=3$.

Figure 4 shows an example of numerical results for $\mathrm{n}=1$ (Newtonian viscosity) and $r=10(10$ units of slip and 1 unit of deformational motion when side drag is absent). All solutions show a marginal boundary layer characterized by strong horizontal shearing that separates interior flows of the slow ridge and fast stream where horizontal gradients are absent or small.

In Figure 4 the boundary layer lacks the reflection symmetry of that for a small step in $\xi$ shown in Figure $3 \mathrm{~b}$. The asymmetry can be understood fairly simply. The local effective value of $r$ is small (large) in the ridge

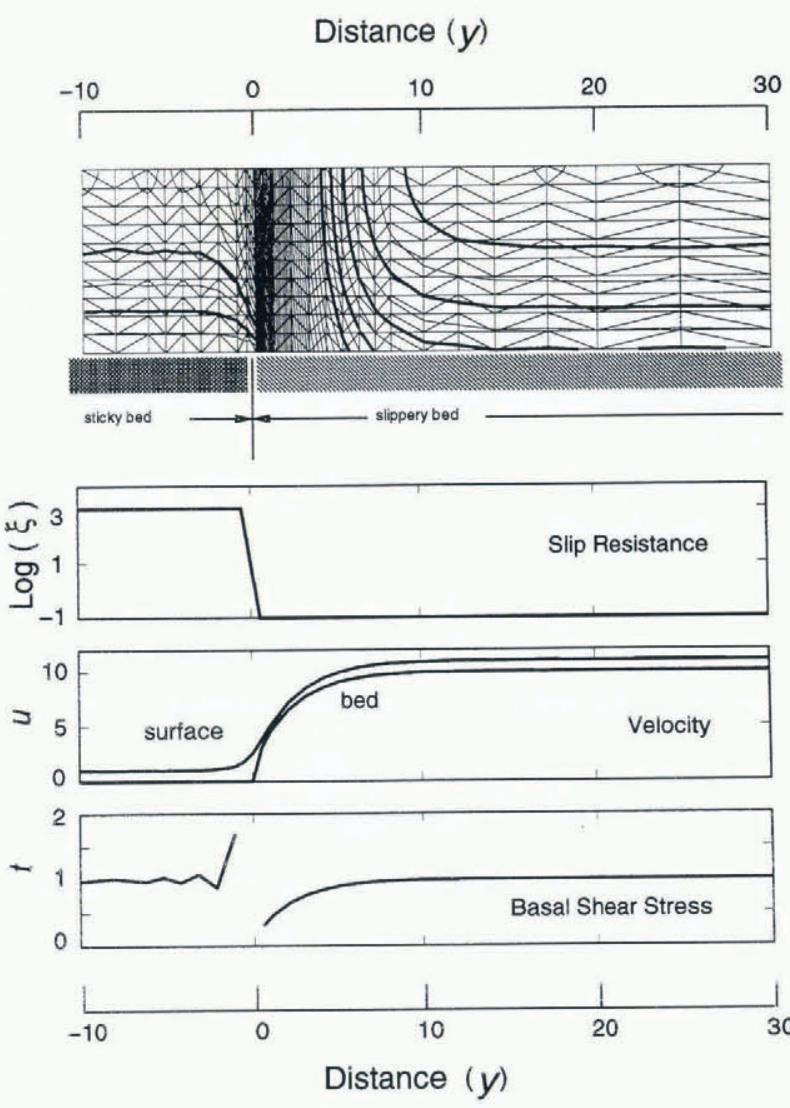

Fig. 4. Finite-element solution for linear-flow and linearslip laws with the shown variation of slip resistance $\xi$. The solution is for $r=10$. Paths passing through triangular elements are contours of constant speed. Every other contour is highlighted in the slow (ridge) and fast (stream) zones. Contours in the shear zone are not highlighted because of the close spacing. 
(stream) yiclding a narrow (broad) stress-concentration (relaxation) zone. The increase in $t_{\mathrm{b}}$ in the stressconcentration zone is much larger than the drop in $t_{b}$ in the stress-relaxation zone, which is expected from the different width scales and global force balance. Because of the essentially no-slip condition at the base under the ridge $(y<0)$, there is no expression of the stressconcentration zone in $u_{\mathrm{h}}$. However, the stress redistribution causes enhanced shearing in the overlying ice, so that the stress-concentration zone affects $u_{\mathrm{s}}$ with a width of about $1[H]$ associated with the transfer $F_{u}$ Equation (28a)). The overall width of the boundary laver is dominated by the stress-relaxation zone with a scale determined primarily by the transfer $F_{\mathrm{sl}}$ (Equation (28c)).

Figure 5 shows the patterns of surface speed $u_{\mathrm{s}}(y)=$ $u(y, 0)$, side-shear strain rate $e(y, 0) \equiv 0.5 \partial u(y, 0) / \partial y$, and basal speed $u_{\mathrm{b}}(y)=u(y, 1)$ for $n=1$ (Fig. 5a-c) and $n=3$ (Fig. 5d-f) with a range of choices for $r$. Comparison of Figure $5 \mathrm{a}-\mathrm{c}$ and Figure $\overline{\mathrm{d}} \mathrm{f}$ shows that the overall width of the boundary layer is narrower for $n=3$ than for $n=1$. The boundary-layer asymmetry and width increase as $r$ increases for both $n=1$ and $n=3$. The side-shear strain-rate disturbance at the surface is spread farther into the ridge i.e. the expression of the stress concentration zone at the surface is broader) for $n=3$ than for $n=1$. This behavior is associated with the strain-rate softening arising from the flow-law nonlinearity that causes stress guiding in a relatively stiff, lowstrain-rate near-surface layer moving over relatively soft, rapidly shearing ice near the base.

The maximum shear strain rate on the surface $e_{\mathrm{m}}$ appears to be just in board $(y>0)$ of the margin $y=0$. For $n=3$ and values of $r$ less than $10^{5}$, the displacement is less than $0.5[H]$, which is the resolution of the numerical calculations. When $r$ exceeds $10^{5}$ a small displacement appears to be resolved, but it remains less than $1[H]$. The value of $e_{\mathrm{m}}$ depends systematically on $r$ Fig. 6a) and can be represented approximately by

$$
2 e_{\mathrm{II}} \equiv \partial u(\approx 0,0) / \partial y \approx r^{n /(1+n)}
$$
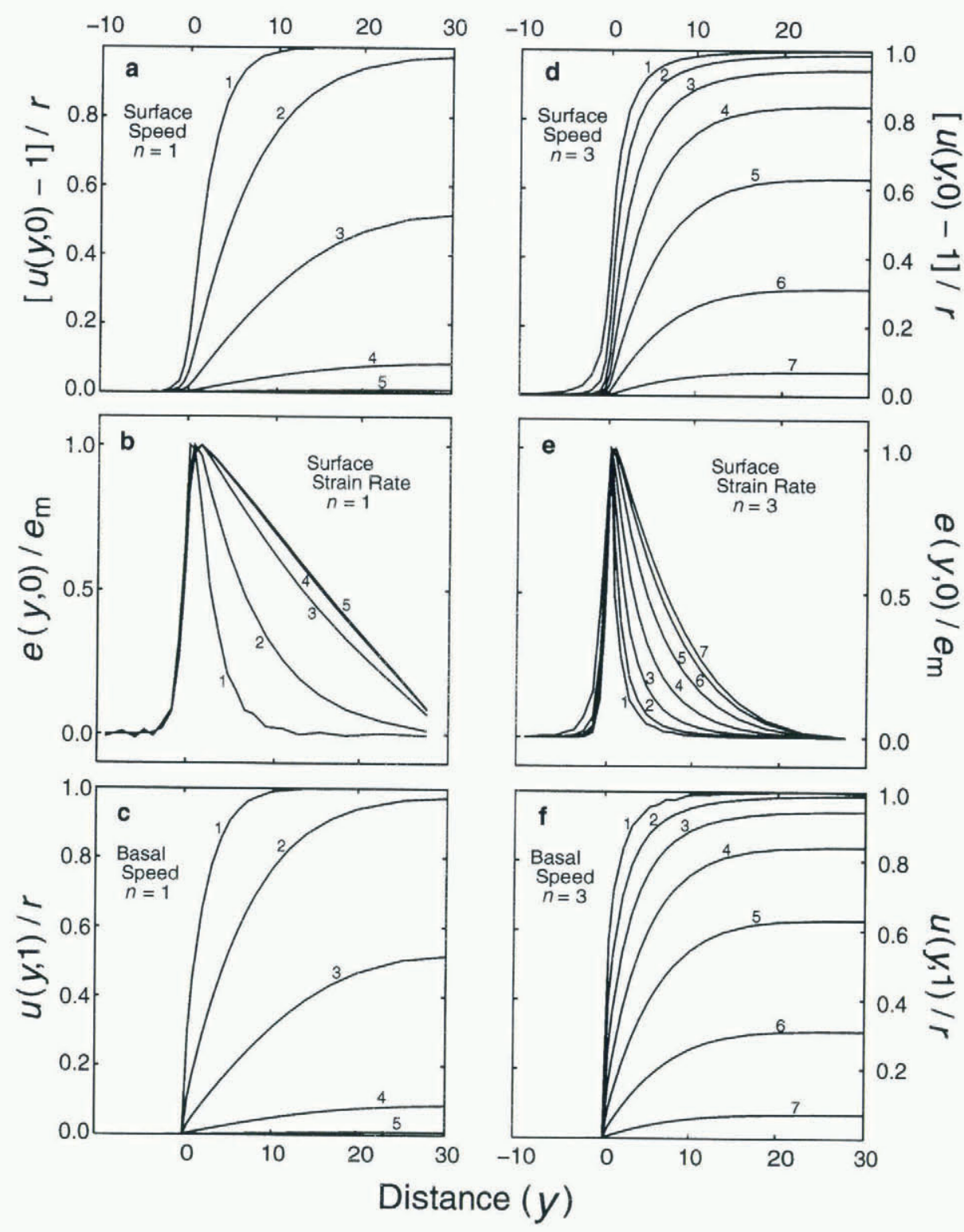

Fig. 5. Distributions of surface speed, surface shear strain rate and basal speed for linear $n=1(a, b$ and $c)$ and power $n=3$ ( $d$, e and $f$ ). flow law wilh selected values of slip ratio $r$. Numbers on curves indicale values of $r$ in powers of 10. Surface shear strain rale in (b) and (e) is normalized by the maximum value $e_{m}$. 

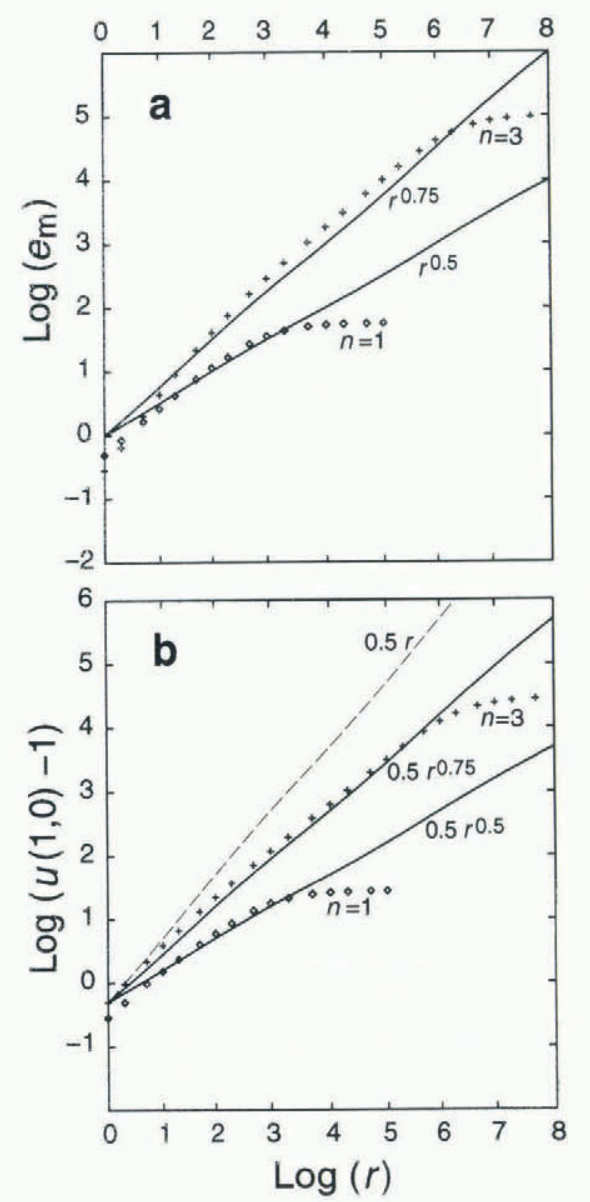

Fig. 6. Dependence of maximum shear strain rate (a) and surface speed at the margin (b) on slip ratio $r$.

for $10^{1}<r<10^{2}$ with $n=1$ and for $10^{1}<r<10^{3}$ with $n=3$. The reason for this dependence becomes clear in the next section.

The surface speed at the margin $u_{s}(0)=u(0,0)$ is related to the pattern of side shearing on the surface. It is smaller than the average of the distant speed on either side of the boundary layer, which is $1+r / 2$ (Fig. $6 \mathrm{~b}$ ). The results determine that approximately

$$
u(0,0)-1 \approx 0.5 r^{n /(1+n)}
$$

for $10^{1}<r<10^{2}$ with $n=1$ and for $10^{1}<r<10^{3}$ with $n=3$.

As $r$ becomes large $\left(>10^{2}\right.$ for $n=1,>10^{3}$ for $\left.n=3\right)$, the boundary-layer width exceeds the half-width of $30[H]$ assumed in the numerical solution (Fig. 5), and the relationships described by Equations (29) and (30) break down (Fig. 6). This behavior arises because the boundary layers from each side bridge across the full stream width. Side drag then affects the full flow width, and speed in the center is less than expected in the absence of side drag (Fig. 5a and d).

\section{Boundary-layer characteristics}

The departure from reflection symmetry, boundary-layer width and bridging are all interrelated. This section quantifies how these characteristics depend on slip ratio $r$. To explore this dependence, I introduce

$$
R_{n}=\left(\frac{r}{n+1}\right)^{\frac{1}{n+1}}
$$

For $n=1, R_{1}=(r / 2)^{0.5}$. For $n=3, R_{3}=(r / 4)^{0.25}$. It turns out to be convenient to compare solutions for different $r$ and $n$ on the basis of $R_{n}$. The rationale for this particular variable is developed in the next section.

There are various alternatives for describing the width $l$ of the boundary layer. For simplicity I use the distribution of $u_{\mathrm{b}}$, and define $l$ to be the distance from the margin $(y=0)$ to the point where $u_{\mathrm{b}}$ reaches 0.8 times the actual speed at the center $u_{\mathrm{b}}(w)$, that is

$$
u_{\mathrm{b}}(l)=0.8 u_{\mathrm{b}}(w)
$$

Reference to $u_{\mathrm{b}}$ does not differ significantly from $u_{\mathrm{s}}$, because of the domination of the width by the stressrelaxation zone and the small difference $\approx 1\left[U_{\mathrm{D}}\right]$ between $u_{\mathrm{s}}$ and $u_{\mathrm{b}}$ for the values of slip ratio $r$ under consideration. The factor 0.8 was chosen with the following considerations. A factor 1.0 is not practical, since the approach of $u_{\mathrm{b}}$, to the limiting value of $r$ is asymptotic. A factor much less than 1 would be sensitive only to the details of shearing very close to the margin.

Figure 7a shows how $l$ is related to $r$ described in terms of $l / w$ and $R_{n} / w$ as found from the numerical calculations with $w=30$ (Fig. 5). For $R_{n} / w<0.1$, the numerical results for $n=1$ and $n=3$ approximately coincide, and for this low range of $R_{n} / w$,

$$
l \approx 1.3 R_{n} \text {. }
$$

Equations (31) and (33) imply that $l$ is proportional to $r^{1 /(n+1)}$. For $n=1, l \approx 0.9 r^{1 / 2}$, which is consistent with the analytical result for small jumps in $\xi$ (Equation (28c)). The corresponding result for $n=3$ is $l \approx 0.9 r^{1 / 4}$. This dependence of $l$ on $r$ implies that the mean sideshear strain rate should scale as $r / l=r^{(n+1) / n}$, which explains the power in the dependence of $e_{\mathrm{m}}$ on $r$ (Equation (29)).

The effect of bridging is illustrated in Figure $7 \mathrm{~b}$, which shows $u_{\mathrm{b}} / r=t_{\mathrm{b}}$ in the center $(\mathrm{y}=\mathrm{w}=30)$. Keep in mind that in the absence of any side drag $\partial t_{y} / \partial y=0, t_{\mathrm{b}}=1$ and $u_{\mathrm{b}}=r$. For small values of $r$ such that $R_{n} / w<0.1$, side drag does not affect $u_{\mathrm{b}}(w)$ and $t_{\mathrm{b}}(w)$. For large values of $r$ such that $R_{n} / w>1, u_{\mathrm{b}}(w)$ and $t_{\mathrm{b}}(w)$ are reduced well below values for no side drag, and the dynamics in the center are dominated by side drag. In the super-high $r$ regime with $R_{n} / w \gg 1$, the stream flow is essentially like an ice shelf where $t_{\mathrm{b}} \approx 0$ and support is entirely by side drag. With $n=3$, side and basal drag are equal for $R_{3} / w=0.5$, which defines a transition between side- and basal-dominated regimes.

\section{GENERALIZATION WITH A SIMPLIFIED MODEL}

\section{Formulation}

To generalize the numerical results to widths other than $30[H]$, I use a one-dimensional boundary-layer model to predict the variation of basal speed $u_{\mathrm{b}}(y)$ under the 

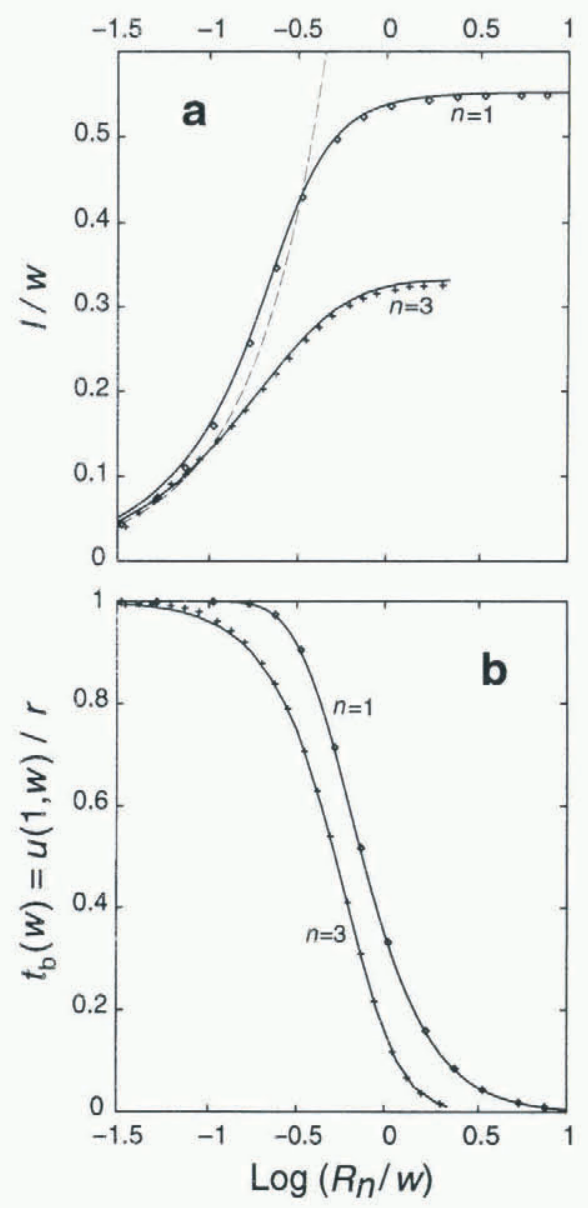

Fig. 7. Dependence of boundary layer width I (a) and center-line basal shear stress $t_{1},(w)=u_{1},(w) / r$ (b) on slip ratio $r$ expressed through $R_{n}$ (Equation (31)) for linear $(n=1)$ and non-linear $(n=3)$. flow lawes. Points show resulls from numerical calculations with $w=30$. Solid curves show resulls from simplified boundary-layer model (Equations (.34), (35) and (11b)). In (a). dashed curve showes Equation (33).

stream flow. The model is based on the flowlaw Equation (9) and conservation of momentum Equation (10) with two assumptions about stress and strain rate at the base $z=1$ : (i) $\partial t_{z} / \partial z=-t_{1}$, and (ii) $\partial u / \partial y \gg \partial u / \partial z$. The lirst means that $\partial t_{z}(y, 1) / \partial z$ is the same as its average over depth $-t_{\mathrm{b}} / 1$. The second is reasonable over much of the width of the boundary layer, which is characterized by dominantly horizontal gradients. It is signilicant only in the case that $n \neq 1$, when $\partial u / \partial z$ affects the calculation of effective strain rate and viscosity. To analyze the onedimensional model I make the substitutions

$$
y=v R_{n} \quad u_{\mathrm{b}}=r \mu .
$$

With assumptions (i) and (ii) and substitutions Equation (34)), Equations (9) and 10 reduce to

$$
\frac{\partial}{\partial v}\left|\frac{\partial \mu}{\partial v}\right|^{\frac{1}{n}} \operatorname{sgn}\left(\frac{\partial \mu}{\partial v}\right)-t_{1}+1=0 .
$$

This equation is to be solved for $\mu$ as a function of $v$ on $\left[0, w / R_{n}\right]$ with the boundary conditions of no motion at the margin $\mu(0)=0$ and no side shear in the center $\partial \mu\left(w / R_{n}\right) / \partial v=0$. When $t_{b}$, depends on $u / r=\mu$ e.g. Equation (11b), Equations (34) and (35) show that $u / r$ is a function of $y / R_{n}$. This is the rationale for the introduction of $R_{n}$ (Equation (31)) and its use in the scaling of axes in Figure 7.

Equation (35) illustrates the underlying cause for a finite-width boundary layer, which stems from the property of the slip law Equation (11b) that $t_{\mathrm{b}}$ increases monotonically with $u_{\mathrm{b}}$. As $u_{\mathrm{b}}$, increases from the margin with distance into the stream flow, $t_{\text {b }}$ also rises. As $u_{\mathrm{l}}$, approaches $r, t_{\mathrm{b}}$ approaches 1 , and side drag must drop to nil.

For $n=1$ and $t_{\mathrm{b}}$, given by Equation (11b) with $\xi(y)=1$ for $y>0$, the solution to Equations (35) and (34) for $u_{\mathrm{b}},(y)$ is straightforwardly found to be

$$
u_{\mathrm{b}}(y)=r\left(1-\cosh \left(\frac{w-y}{\sqrt{r / 2}}\right) / \cosh \left(\frac{w}{\sqrt{r / 2}}\right)\right) .
$$

For $n=3$, I have not succeeded in finding a useful analytic representation of the solution to Equation (35). I therefore resorted to numerical integration to explore characteristics of its solutions.

\section{Test against full numerical model}

The predictions of the one-dimensional model for the dependence of $l$ and $u(w)$ on $r$ and $w$ as expressed through $R_{n} / w$ are shown in Figure 7 , where they are compared to the numerical results from the full equations for $w=30$. In spite of the assumptions, the onedimensional model predicts the boundary-layer width and the onset of bridging very accurately. The difference between the simplified and full numerical model is most evident for small values of $r$, where both calculations begin to break down from lack of resolution when the boundary laver gets narrow in the case of the full numerical calculations or of the failure of assumptions i) and (ii) in the simplified model. Except for this restriction, the numerical results in Figure 7 can then be generalized to $w \neq 30$.

Figure 8 shows the shape of the cross-profile of speed predicted by the one-dimensional model for $n=3$, when there is no significant bridging calculated for $w / R_{n}=20$, vielding $t_{1},(w)=0.99$ ). The results from the numerical calculations for which there was no significant bridging

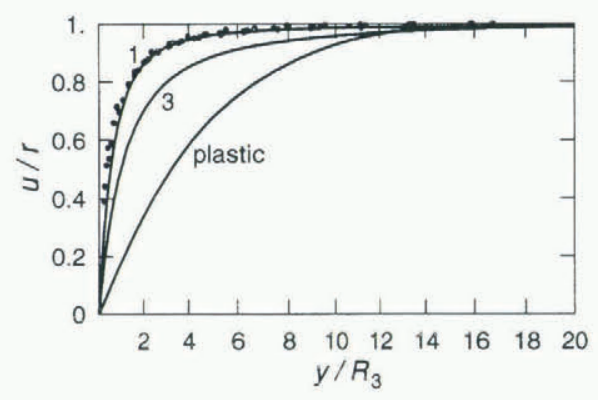

Fig. 8. Basal speed versus distance for different basal slip lawes (Equations (36) and (37)). Numbers on curves gize power $m$ in Equation (36). Points give results from numerical calculations for $n=3$ and $r=10,20,50,100$. 
$(r=10,20,50,100)$ are also plotted in Figure 8 as points. The points represent $u_{\mathrm{b}} / r$ calculated at grid points with $y$ scaled by $R_{3}$. With this rescaling of $y$, the distinct curves of $u_{\mathrm{b}},(y)$ in Figure 5 collapse on to approximately a single curve that describes the boundary-layer structure.

Comparison of the one-dimensional model and the full numerical model shows a small, systematic difference near the margin. It arises because the ice directly over the margin $(y=0)$ in the full model is free to move (e.g. $u_{\mathrm{s}}$ in Figure 5), whereas $u=0$ is implicitly imposed by the assumptions of the one-dimensional model. Although the agreement is not perfect, it is quite good.

In Figure 8, one can see that the width of the boundary layer $l$ (Equation (32)) is about 1.3 in units of $R_{3}$ in accordance with Equation (33). It also illustrates the extreme plug-like flow associated with a narrow boundary layer introduced by a velocity-dependent drag at the base.

\section{Non-linear slip}

The precision of the one-dimensional model suggests that it can be used to explore the implications of non-linearity in the slip condition. For this purpose, I assume a slip law of the form

$$
t_{\mathrm{b}}=\left(\frac{u_{\mathrm{b}}}{r}\right)^{\frac{1}{m}}
$$

When $m=1$, Equation (37) is equivalent to the slip law used carlier (Equation (11b)). When $m=2$, it gives the dependence $u_{\mathrm{b}} \propto t_{\mathrm{b}}{ }^{2}$ commonly assumed for sliding over hard beds as originally proposed by Weertman (1957) on the basis of motion past scaleless bed roughness by a combination of creep and regelation. If there are only large roughness elements present, then $m=3$ is indicated. Equation (37) preserves the condition that $t_{\mathrm{b}}=1$ when $u_{\mathrm{b}}=r$.

$\mathrm{I}$ also consider a basal-yield condition such that

$$
u_{\mathrm{b}}=0, t_{\mathrm{b}}<t_{0} \quad t_{\mathrm{b}}=t_{0}, u_{\mathrm{b}}>0 .
$$

This slip law is an extreme non-linear version of Equation (37) and would approximate slip over a perfectly plastic subglacial material with a distinct vield stress $t_{0}$. The glacial till beneath ice streams by itself could have a nearperfectly plastic behavior (Kamb, 1991). Under the stream where $u_{\mathrm{b}} \neq 0$, Equation (38) requires that $0<t_{0}<1$ and $t_{\mathrm{b}}=t_{0}$ everywhere. Correspondingly, the support of all ice columns by side drag is the same $\left(1-t_{0}\right)$. The solution for this plastic slip law in the simplified model is easily derived by integration of Equation (35) with $t_{\mathrm{b}}=t_{0}$ and substitution back into Equation (34), which gives

$$
u_{\mathrm{b}}(y)=\left(1-t_{0}\right)^{n} w^{n+1}\left[1-(1-y / w)^{n+1}\right] .
$$

For this solution, the boundary-layer width $l$ defined by Equation $(32)$ is $l / w=0.55$ for $n=1$ and $l / w=0.33$ for $n=3$. These values predict the limiting values of $l / w$ for very large $r$ (Fig. 7a), for which $t_{\mathrm{b}}$ approaches zero everywhere under the stream flow (Fig. 7b).

Figure 8 compares the variation of $u_{\mathrm{b}}$ found for $n=3$ and $w / R_{n}=20$ using $m=1$ and 3 in Equation (37). A profile for $u_{\mathrm{b}}$ predicted from Equation (38) is also shown for the case that $w$ and $t_{0}$ are adjusted to give the same $u_{\mathrm{b}}(w)$.

The results in Figure 8 show that the boundary layer is broader for the non-linear slip laws compared to the linear one. The non-linearity $(m>1)$ makes it difficult to raise $t_{\mathrm{b}}$ to $1[T]$. In the case of a basal-yield condition (Equation (38)) with $t_{0}<1$, it is not possible to raise $t_{\mathrm{b}}$ to $1[T]$ for any $u_{\mathrm{b}}$. An alternative viewpoint is that the nonlinearity in the slip law results in a smeared-out variation of an effective slip resistance that broadens the boundary layer.

\section{DISCUSSION}

\section{Comparison with observations}

The spatial scale of spreading $l$ should be apparent in observations of cross-variation of velocity in ice streams. The theoretical size of $l$ can be predicted from Equations (8), 31 and $(33)$, when deformational speed $U_{\mathrm{D}}$ can be calculated (Equation (7)) and basal speed can be backed out from velocity measurements Equation (6c)). The direct expression of $l$ in observations can be found from measurements of velocity through the marginal shear zones and Equation (32).

For the Siple Coast ice streams, the thickness, driving stress and central surface speed have typical values: $H \approx 10^{0} \mathrm{~km}, T \approx 10^{1} \mathrm{kPa}$ or less and $\hat{u}_{\mathrm{s}}(\hat{w}) \approx 10^{2} \mathrm{ma}^{-1}$ or more. On the basis of a flow law (Paterson, 1994, table 5.2 evaluated for the vertically averaged ice temperature of $-15 \mathrm{C}$ (Engelhardt and others, 1990), Equation (7b) predicts a deformational speed $U_{\mathrm{D}}$ of order $10^{-1} \mathrm{ma}^{-1}$ or less. Comparison of $U_{\mathrm{D}}$ to $\hat{u}_{\mathrm{s}}$ indicates that $u_{\mathrm{s}}$ and $u_{\mathrm{b}}$ are essentially the same and are order $10^{4}$ or more. The slip ratio $r$, which is the bed speed in the absence of side drag, is then order $10^{4}$ or larger when allowance is made for the reduction in actual speed by side drag (Fig. 7b). Equations (31) and (33) then predict $R_{3} \approx 7$ or more and $l \approx 10$ or more. The corresponding dimensional scale of spreading introduced by thickness $H$ is then $\hat{l} \approx 10 \mathrm{~km}$ or more. An implication of these evaluations is that lateral variations in $u_{\mathrm{s}}$ on these ice streams should occur at scales that are about $10 \mathrm{~km}$ or longer.

In some places on the margins of Siple Coast ice streams, the shear zones are wider than $\hat{l} \approx 10 \mathrm{~km}$. Examples have been found in Ice Streams E (Bindschadler and Scambos, 1991, fig. 6), B2 (Whillans and others, 1993, upstream part of transect), and D (Scambos and others, 1994, profile 5). A wide shear zone can be explained by laterally distributed variations in the slip resistance arising from spatial changes in variables that control $\xi$. Slip-law non-linearity could also contribute to a gradational variation of an effective slip resistance (Fig. 8). Indeed, the irregularity of the surface velocity variations found on Ice Stream E indicates that similarly complex variations occur in the basal-boundary condition Bindschadler and Scambos, 1991; MacAyeal, 1992). Extreme examples are locales within a broader marginal zone of increasing speed where the cross-profile of speed is concave toward the down-flow direction and the ice 
motion is actually being dragged forward rather than being resisted from the side. Thus, from the point of view of side drag alone, the interior ice is isolated from the drag from the margins and instead responds to more local influences from the side (and probably along the length). These kinds of observation show that the change from sticky under a ridge to slippery under an ice stream can be transitional and irregular.

In other places on the margins of Siple Coast ice streams, the marginal shear is concentrated in zones that are narrow compared to $\hat{l} \approx 10 \mathrm{~km}$. Examples have been found in Ice Streams B Bindschalder and others, 1987, DwnB; Whillans and others, 1993, downstream part of B2 transect; Echelmeyer and others, 1994, UpB), D Scambos and others, 1994, profile 1) and E (Bindschadler and Scambos, 1991). The boundary-layer widths as defined from Equation 32 are $1.4 \mathrm{~km}$ at $\mathrm{LpB}, 4 \mathrm{~km}$ at $\mathrm{D} l$ and $5 \mathrm{~km}$ at DwnB. The distortion of crevasses in some locations points to bands of shearing as narrow as $1 \mathrm{~km}$ Merry and Whillans, 1993, fig. 3).

Within the context of this analysis, such narrow shear zones would require values of $r$ that are much smaller than could be consistent with the measured $u_{\mathrm{s}}$ and $U_{\mathrm{D}}$ expected from the normal flow law for ice. The discrepancy is very large at $\mathrm{UpB}$, which is seen straightforwardly from the observations of Echelmeyer and others (1994): $H=1.1 \mathrm{~km}, \hat{w} \approx 14 \mathrm{~km}$ and $\hat{l} \approx$ $1.4 \mathrm{~km}$. By reference to Equation 32$)$, the last is estimated as the lateral distance between locations on the upper surface of maximum shear strain rate and $80 \%$ of center speed, which utilizes the expectations that the margin (i.e. the location of a jump in slip resistance at the bed lies beneath the maximum in shear strain rate and that the surface and bed speeds in the ice stream are nearly the same. It follows that $l \approx 1.3 . l / w \approx 0.1$. Figure $7 \mathrm{a}$ and Equation (31) then imply that $R_{3}=1$ and $r=5$, which is orders of magnitude too small given the measured $\hat{u}_{\mathrm{s}}(\hat{w})=0.43 \mathrm{~km} \mathrm{a}^{1}$ and any reasonable $U_{\mathrm{D}}$. The situation is not so extreme for DI where the same reasoning leads to $l / w \approx 0.26$, and the implied values for $R_{3} \approx 6$ and $r \approx 5 \times 10^{3}$ are closer to the range of possibility $\left(r>10^{1}\right)$ but still distinctly too low.

Echelmeyer and others (1994) and Scambos and others 1994) show that these narrow shear zones can be explained by a localized softening (shear enhancement) of the ice that localizes the shearing. However, it is important to recognize that localization of shear at scales less than order 10 ice thicknesses does not, in general, prove that there is local softening of the ice. That is only the case when $r$ is high slip much larger than deformation ).

\section{Implications for inversions}

The above discussion motivates consideration of the inverse problem of deducing the distribution of slip resistance $\xi$ from measurements of surface speed $u_{\mathrm{s}}$. Realistic inversions of measurements should account for both longitudinal and lateral variations in slip conditions and non-linearity of the ice-flow law (MacAyeal, 1992). It also seems important to include variations in ice viscosity arising from fabric and/or temperature Echelmeyer and others, 1994). Nevertheless, some specific problems associated with inversion arising from lateral variations can be identified based on the general analysis for a linear flow law with small variations in $\xi$. It is useful to consider the inversion as a two-step process: first, determine $u_{\mathrm{b}}$ from $u_{\mathrm{s}}$ (Equation (2la)) ; second, determine $\xi$ from $u_{\mathrm{b}}$, Equation $\left.24 \mathrm{a}\right)$ ), which in combination are expressed as Equation $25 \mathrm{c}$ ).

The first step is illustrated by Equations (21) and Figure 2. High frequency $\left(\nu>1\left[H^{-1}\right]\right)$ variations in $u_{\mathrm{b}}$ are strongly attenuated $\left.\tilde{F}_{11} \approx 0\right)$ at the free upper surface. Thus, within the physics of the model, any high-frequency fluctuations in $u_{\mathrm{s}}$, however small, would have to come from very large fluctuations in $u_{\mathrm{b}}$. The calculation of $t_{\mathrm{b}}$ from $u_{\mathrm{s}}$ is similarly unstable Equations (23) and (21)). Noise in $u_{\mathrm{s}}$ from measurement errors or inadequacy of the physical model, such as shear enhancement of the type discussed above, would tend to obscure any actual signal associated with the bed, and make accurate inversion for $u_{\mathrm{b}}$ and $t_{\mathrm{b}}$ at spatial scales smaller than $1[H]$ hopeless (Fig. 2). The length $1[H]$ then separates two spatial scales: long $\gg 1[H]\left(F_{\mathrm{u}} \approx 1\right.$ in Equations $\left.(21)\right)$ and short $<1[H]\left(F_{\mathrm{u}} \approx 0\right.$ in Equations 21$)$ ) for which inversion is practical or not.

The behavior for lateral variations in $u$ has some similarity to features known for longitudinal variations (c.g. Balise and Raymond, 1985), but it is fortunately less complex. For longitudinal variations, there is an intermediate scale for which surface and basal variations in $u$ have opposite sign. There is no corresponding behavior for the lateral variations. The differences arise largely because longitudinal variations involve blocking along flow paths that cause interactions imposed by continuity. Lateral variations interact by shear alone and are free of such continuity constraints.

The second step is motivated by the realization that variations in $u_{\mathrm{b}}$, arise from variations in $\xi$, and therefore may be expected to have certain properties. In the high $r$ range (high mean basal slip), a localized disturbance in $\xi$ is converted into a relatively broader disturbance in $u_{1}$, and $t_{\mathrm{b}}$, Equations (24) and 25); Figs 2 and 3 with a width $l \gg 1[H]$ ). Furthermore, even though the variations in $\xi$ and $u_{4}$, may be small compared to their respective means, the long-scale variations in $u_{3}$, can be much larger than the deformational speed $U_{\mathrm{D}}$.

Now consider both steps in combination for high $r$. Induced variations $u_{\text {}}$, will have long spatial-scale features. At this scale, they can be transferred to the surface with only minor attenuation to appear as a signal in $u_{\mathrm{s}}$. Moreover, the transfer from the bed to the surface is not attenuated by a depth-varying speed (e.g. Figs 2 and 3, Equations $\left(21\right.$ ) with $F_{11} \approx 1$ ). These results then support inversion for slip resistance at long spatial scales with a depth-integrated model such as used by MacAyeal (1992). However, inversion for short-scale features in slip resistance is confounded by spatial spreading both over the bed and through the ice that smooths their effects at the location of potential observation on the upper surface.

A corollary of this discussion is that any substantial lateral variation of $u_{\mathrm{s}}$ with a scale much shorter than $l$ must arise from physics that is not included in the model. for example, short-scale, lateral variations in the properties of the ice.

Although the above discussion is cast in terms of the 
theory for a linear fluid, the numerical calculations show the same features occur for a power-law fluid like ice with the quantitative difference that the spreading is mediated by the scale $R_{n}$ (Equation (31)) with $n=3$ (Figs 7 and 8 ).

\section{CONCLUSIONS}

The central conclusion is that a local, cross-flow (lateral) variation in basal slip resistance $\xi(y)$ has a non-local effect on basal speed $u_{\mathrm{b}}(y)$ and stress $t_{\mathrm{b}}(y)$. The scale of the lateral spreading $l$ in relation to ice thickness is determined by $R_{n}$ (Equations (31) and (33)), which increases as as a power of the ratio of basal slip to internal deformation. Within the zone of spreading, the driving stress is balanced by a combination of basal and side drag. The distributions of $t_{\mathrm{b}}(y)$ and $u_{\mathrm{b}}(y)$ are positively and negatively, respectively, correlated with $\xi(y)$.

In the case of a shear margin originating from a jump in $\xi$ from very high to low values, this general result is manifested as a boundary layer with a width $l$ (Equation (32)) that extends into the fast-slipping (low $\xi$ ) side of the boundary. Reduction of basal drag on the fast-slipping side of the boundary is concentrated in a zone of high stress with a width of about one thickness on the slowslipping (high $\xi$ ) side.

Three regimes of side drag can be identified for an ice stream of half-width $w$. For $R_{n}<0.1 w(l<0.13 w)$, side drag is restricted to the sides and does not affect the central part of an ice stream. For $R_{n}>1 w(l \rightarrow 0.33 w)$, side drag dominates and $t_{\mathrm{b}} \rightarrow 0$ even in the center. Siple Coast ice streams are in an intermediate regime with driving stress in the center balanced by a combination of side and basal drag. Relative to predictions of the analysis, localized softening of the ice in the shear margins of these ice streams causes boundaries that are more narrow, and results in less side drag in the interior.

\section{ACKNOWLEDGEMENTS}

This research was supported by grant No. DPP9118703 from the U.S. National Science Foundation. Comments from D. MacAyeal stimulated many improvements in the presentation of the paper.

\section{REFERENCES}

Balise, M.J. and C.F. Raymond. 1985. Transfer of basal sliding variations to the surface of a linearly viscous glacier. f. Glaciol., 31 109) 308-318.

Batchelor, G. K. 1967. An introduction to fluid dynamics. Cambridge, etc., Cambridge Lniversity Press.

Bentley, C. R. 1987. Antarctic ice streams: a review. J. Geoplys. Res., 92 B9 , 8843-8858.

Bindschadler. R. A. and T. A. Scambos. 1991. Satellite-image-derived velocity field of an Antarctic ice stream. Science, 252 5003, 242246.

Bindschadler, R. A., S. N. Stephenson, D. R. Mac.lyeal and S, Shabtaie. 1987. Ice dynamics at the mouth of Ice Stream B. Antarctica. J. Geophys. Res., 92 B9), 8885-8894.

Bracewell, R.N. 1978. The Fourier transform and its applications. Second edition. New York. McGraw Hill.

Echelmeyer, K. A., W. D. Harrison, C. Larsen and J. E. Mitchell. 1994. The role of the margins in the dynamics of an active ice stream. $\bar{f}$. Glaciol., 40 136), 527-538.

Engelhardt H., N. Humphrey, B. Kamb and M. Fahnestock. 1990. Physical conditions at the base of a fast moving Antarctic ice stream. Science, 248 4951), 5759.

Harbor. J. M. 1992. Application of a general sliding law to simulating flow in a glacier cross-section. f. Glaciol.. 38 128, 182 90.

Kamb, W. B. 1991. Rheological nonlinearity and flow instability in the deforming bed mechanism of ice stream motion. \%. Geophys. Res., 96 В $10,16.585 \quad 16.595$.

MacAyeal, D. R. 1992. The basal stress distribution of Ice Stream E, Antarctica, inferred by control methods. \%. Geophys. Res., 97 B1). $595-603$.

Merry, C.J and I. M. Whillans. 1993. Ice-flow features on Ice Stream B. Antaretica, revealed by SPOT HRV imagery. 7. Glaciol., 39 133). $515-552$.

Nye, J. F. 1965. The flow of a glacier in a channel of rectangular, elliptic or parabolic cross-section. J. Glaciol., 5 41), 661-90.

Paterson, W. S. B. 1994. The physies of glaciers. Third edition. Oxford, etc., Elsevier.

Raymond, C. F. 1971. Flow in a transverse section of the Athabasca Glacier, Alberta, Canada. J. Glaciol., 1058 , 5584.

Revnaud. L. 1973. Flow of a valley glacier with a solid friction law. $f$. Glaciol., $1265,251-258$.

Scambos T.A., K.A. Echelmeyer, M.A. Fahnestock and R.A. Bindschadler. 1994. Development of enhanced ice flow at the southern margin of Ice Stream D, Antarctica. Amm. Gilaciol., 20. $313-318$.

Shabtaie, S. and C.R. Bentley. 1987. West Antarctic ice streams draining into the Ross Ice Shelf: configuration and mass balance. $\mathcal{J}$. Geophys. Res., 92 B2), 1311-1336.

Weertman, J. 1957. On the sliding of glaciers. J. Glaciol., 321 , , $33-38$.

Whillans, I. M., M. Jackson and Y.-H. Tseng. 1993. Velocity pattern in a transect across Ice Stream B. Antarctica. J. Glaciol., 39 133), 562 572 . 